\section{Electromagnetic Wave Propagation and Wave-Vector Diagram in Space-Time Periodic Media}

\section{CHARLES ELACHI}

Abstract-The electromagnetic wave propagation and wavevector diagram in generally space-time periodic dielectric, plasma, and uniaxial plasma are studied for $T E$ and TM waves. The case of a sinusoidal periodicity is solved numerically. Special properties due to the inhomogeneity are presented.

\section{INTRODUCTION}

Considerable interest has recently been stimulated by phenomena that involve the interaction between two waves in the presence of liquids, solids, or plasma, including such applications as parametric processes in nonlinear media, diffraction of light by ultrasonic waves, ionospheric perturbations induced by strong electromagnetic signals, and others. These processes usually involve a stronger wave of the electromagnetic, elastic, or other type which modulates the properties of a medium in a space-time periodic manner (i.e., $\alpha(z, t)=\alpha_{0}\left[1+\alpha_{1} f(K z-\Omega t)\right]$, where $f(\xi)$ is a periodic function), which in turn causes a diffraction of the weaker wave, usually of the electromagnetic type. The limit case $\Omega=0$ of a periadically stratified medium is also of great interest.

TE and guided-wave propagation in sinusoidally space-time periodic isotropic media was studied extensively by many authors $[1]-[3]$. The purpose of this investigation is to solve the problem of $\mathrm{TE}$ and $\mathrm{TM}$ waves in generally space-time periodic media (dielectric, plasma, and uniaxial plasma) and to derive the corresponding wave-vector diagrams which are of importance in studies related to dipole radiation [4], [5], propagation in waveguides and interaction with a half-space.

\section{General Solotion}

Let us consider a medium submitted to a strong pump wave which modulates its characteristics as

$$
\alpha(z, t)=\alpha_{0}\left[1+\alpha_{1} f(K z-\Omega t)\right]
$$

where

$\Omega, K$ angular frequency and wavenumber of the fluctuation.

$f(\xi)$ periodic function $=\sum_{m=-\infty}^{m=+\infty} a_{m} \exp (i m \xi)$.

$\alpha(z, t)$ plasma density $N(z, t)$, or dielectric constant $\epsilon(z, t)$ depending on the medium under consideration.

For the plasma case we define the vector $A=\partial E / \partial t$, and for the uniaxial plasma, the static magnetic field is supposed parallel to the $z$ axis $\left(B_{0}=B_{0} e_{z}\right.$ with $\left.B_{0} \rightarrow \infty\right)$.

Using Floquet's theorem, the solution for the electromagnetic field can be written as

$V=\left\{\begin{array}{r}e_{y} \sum_{n=-\infty}^{+\infty} V_{n} \exp [i \delta x+i(\kappa+n K) z-i(\omega+n \Omega) t], \\ \text { for TE waves: } E=E e_{y} \\ \sum_{n=-\infty}^{+\infty}\left(V_{n x} e_{x}+V_{n z} e_{z}\right) \exp [i \delta x+i(\kappa+n K) z-i(\omega+n \Omega) t] \\ \text { for TM waves: } E=E_{x} e_{x}+E_{z} e_{z}\end{array}\right.$

Manuscript received December 1, 1971; revised January 24, 1972 This work was supported in part by NASA under Contract NAS7-100 and in part by the U. S. Air Force under Grant AFOSR-68-1400.

The author is with Jet Propulsion Laboratory, California Institute of Technology, Pasadena, Calif. 91109. where $\boldsymbol{V}$ is defined in Table I and the index $n$ corresponds to the $n$th harmonic generated by the interaction with the modulated medium. $\delta$ and $\kappa+n K$ are the components of the wave vector.

Putting the expression of $V$ in the corresponding Maxwell's and linearized force equations, replacing $f(\xi)$ by its Fourier series, and equating the terms with the same frequencies, we find the following infinite systems of equations:

$\mathrm{TE}$ wave in a dielectric or isotropic plasma

$$
D_{n} V_{n}+2 \sum_{l=-\infty}^{l=+\infty} a_{n-l} V_{l}=0
$$

TM wave in a uniaxial plasma

$$
D_{n} V_{n z}+2 \sum_{l=-\infty}^{l-+\infty} a_{n-l} V_{l z}=0
$$

TM wave in a dielectric or isotropic plasma

$$
\begin{aligned}
& B_{n} V_{n x}+2 \sum_{l=-\infty}^{l-\infty} a_{n-l} V_{l x}+G_{n} V_{n z}=0 \\
& B_{n}{ }^{\prime} V_{n z}+2 \sum_{l=-\infty}^{l-+\infty} a_{n-l} V_{l z}+G_{n} V_{n x}=0
\end{aligned}
$$

where all the terms are defined in Table $I$. These systems of equations can be written in a matrix form $\|M\| \cdot|V|=0$ and the dispersion equation is the nontriviality condition

$$
\Delta(\delta, \kappa, \omega)=\operatorname{det}\|M\|=0
$$

$\|M\|$ is an infinite matrix defined from (2), (3), or (4), and $|V|$ is an infinite column vector with elements $V_{n}$ or $V_{n z}$. The solution of (5) gives the Brillouin and wave-vector diagram $(\delta(\kappa)$ for fixed $\omega)$. This equation is usually solved numerically as we will do later for a sinusoidal periodicity, but to have an insight on the diagrams without any computations we study the limit $\alpha_{1} \rightarrow 0$.

At the limit $\alpha_{1} \rightarrow 0$, equations (2), (3) and (4) reduce to

$$
\alpha_{1} D_{n}=0 \text { or } B_{n} B_{n}^{\prime}=G_{n}^{2} \text {. }
$$

Replacing each term by its expression, we find that the Brillouin diagram is (see Table I) : a family of circles with a straight line envelope for the dielectric and parabolic envelope for the plasma, and a family of conies (hyperbolas or/and ellipses depending on the relative values of $\omega_{n}$ and $\omega_{p o}$ ) for a uniaxial plasma.

These diagrams are shown by the dashed lines on Figs. 1 and 2 (see [4] and [5] for the diagrams corresponding to other cases). For $\alpha_{1} \neq 0$, strong interactions occur at the intersection points giving regions where the solution is complex $\kappa=\kappa_{r}+i_{\kappa_{i}}$. The solution near these regions is largely dependent on the function $f(\xi)$.

From the wave-vector diagrams we see that the solution is multivalued. For a fixed value of $\delta$ there are many solutions $k=\kappa_{a}$ $(q=0, \pm 1, \cdots)$. Therefore, using the principle of superposition, the expression of $V$ must be

$$
\begin{aligned}
V=\sum_{q^{\alpha-\infty}}^{q=+\infty} \sum_{n=-\infty}^{n=+\infty}\left(V_{n q z} e_{x}+\right. & \left.V_{n g z} e_{z}\right) \\
& \cdot \exp \left[i \delta x+i\left(\kappa_{q}+n K\right) z-i(\omega+n \Omega) t\right]
\end{aligned}
$$

and the same summation must be applied in (1). The index $q$ represents the modes.

The relative amplitudes $V_{n q} / V_{o q}, V_{n q z} / V_{o q z}$, and $V_{n q x} / V_{o q z}$ can be determined from (2), (3), or (4), or their matrix form. The reference values $V_{o q}, V_{o q z}$ are determined by the boundary conditions for the half-space problem [4] or the source condition for a dipole or a moving charge [4], [5]

In the dielectric case, the solution of the system in (2) or (4) diverges in a certain region of the values of $\Omega / K$. This region is 
TABLE I

Expression of Parameters

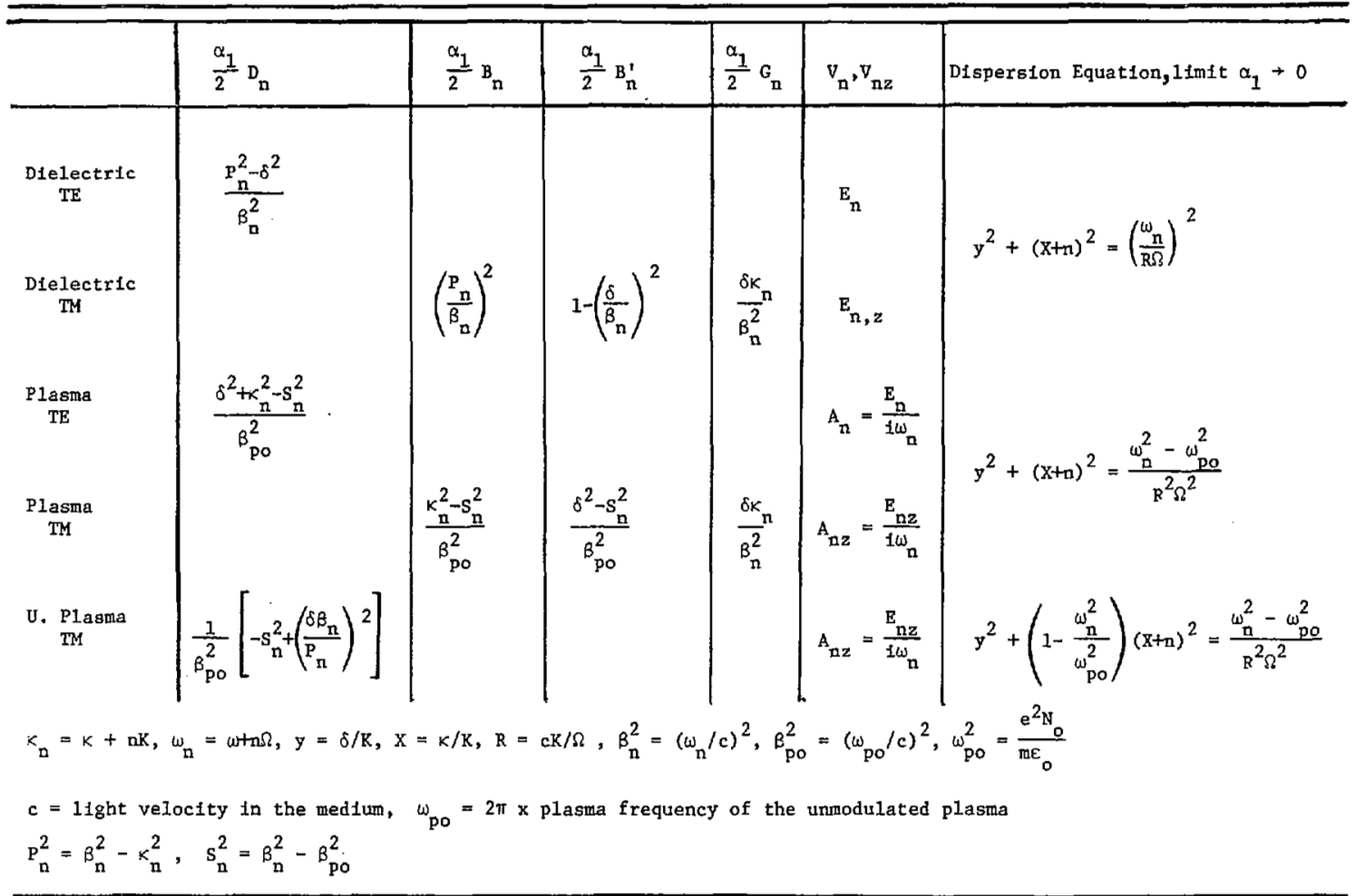

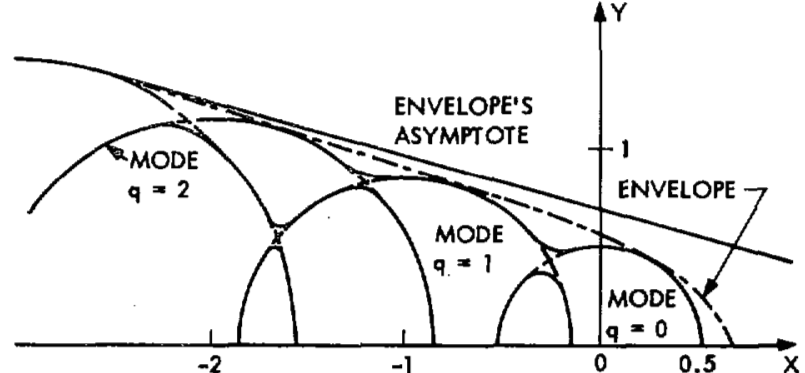

Fig. 1. Wave-vector diagram for a sinusoidally STP plasma: $\omega_{p o} / \omega=0.75, R=3.5, \omega / \Omega=2.5, \alpha_{1}=0.25$ (the dashed line corresponds to $\alpha_{1} \rightarrow 0$ and $f(\xi)$ arbitrary).

called the sonic region [2] and can be determined from Poincare theorem [6]. In a plasma [1] or uniaxial plasma [4] there is no sonic region.

\section{Case of Sinusoidal Periodicity}

We solved the different dispersion equations numerically for the case where $f(\xi)=\cos \xi$ (i.e., $a_{ \pm 1}=\frac{1}{2}$ and $a_{m}=0$ for the rest). The infinite matrix $\|M\|$ was truncated (a $9 \times 9$ matrix gave exact results to the third order). The results are shown in Figs. 1 and 2 (see [4], [5] for other cases). Some of the characteristics of electromagnetic wave propagation in space-time periodic media are as follows.

1) Even if $\omega<\omega_{p 0}$ in a plasma, there is propagation of the higher harmonics.

2) In a stratified medium ( $\Omega=0$ ), for a fixed $\delta$, all the solutions $\kappa_{q}$ are of the same nature: complex, real, or imaginary. If $\Omega \neq 0$, the solutions $\kappa_{q}$ can be of different nature depending on the mode $q$.

3) There are inflection points in the wave-vector diagram. This leads to caustic surfaces when the source is localized [4], [5].

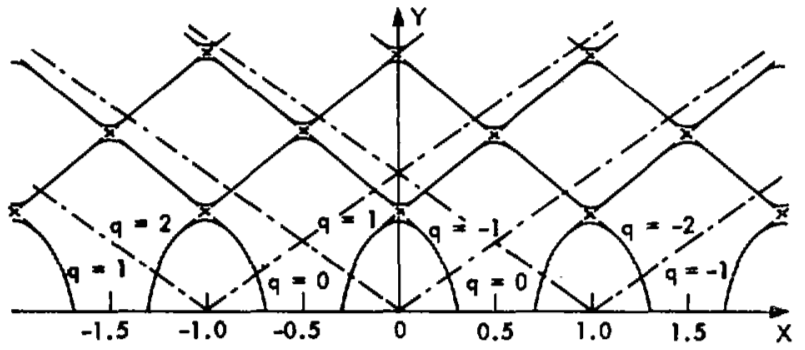

(a)

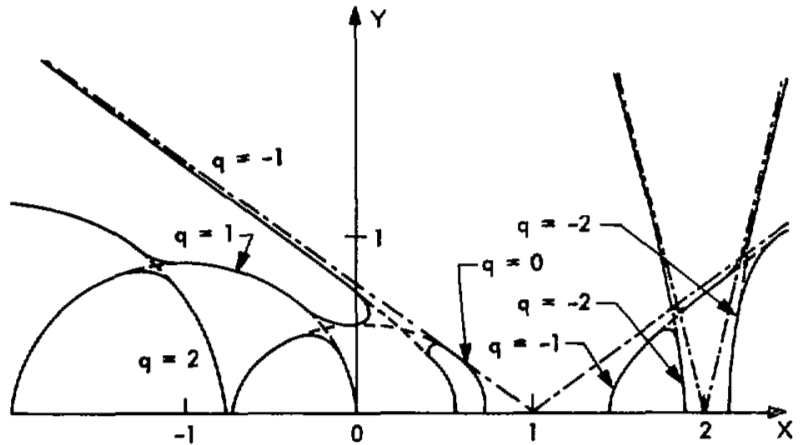

(b)

Fig. 2. Wave-vector diagram for a uniaxial plasma. (a) Sinusoidally stratifled: $\omega_{p o} / \omega=1.25, \alpha_{1}=0.25, c K / \omega=1.4$. (b) Sinusoidally STP: $R=3.5, \omega / \Omega=2.5, \omega_{p o} / \omega=0.75, \alpha_{1}=0.25$ (the dashed lin corresponds to $\alpha_{1} \rightarrow 0$ and $f(\xi)$ arbitrary). 
4) In an homogeneous uniaxial plasma, for $\omega<\omega_{p 0}$ there is group propagation only inside a cone of axis $z$ and half-angle $\theta_{c}=\arcsin \left(\omega / \omega_{p 0}\right)$. In fact, the group angle is given by $\theta_{g}=$ arc $\cot \left(d \delta / d_{\kappa}\right)$ and for $\alpha_{1}=0$ the wave-vector diagram slope is

$$
\mid \text { slope } \mid>\left(\frac{\omega_{p 0}{ }^{2}}{\omega^{2}}-1\right)^{1 / 2} \text {. }
$$

For $\alpha_{1} \neq 0$, the slope near the interaction regions goes to zero. This allows propagation for all angles $\theta$. This effect is caused by the reflections from the inhomogeneities in the plasma density [4], [5].

\section{Conclusion}

The wave-vector diagrams in the limit $\alpha_{1} \rightarrow 0$ are the same for any kind of periodicity $f(\xi)$ and depend on the medium and the parameters $K, \Omega$. The dependence on $f(\xi)$ and the wave polarization ( $\mathrm{TE}$ or $\mathrm{TM}$ ) appears mostly near the interaction regions when $\alpha_{1} \neq 0$.

\section{ACKNowledgment}

The author deeply thanks Prof. C. H. Papas for many helpful discussions during this study.

\section{REFERENCES} [1] J. Askne, "Ware reflection from and propagation in a pumped Sxmp. on Eilectromagnetic Waves.

[2] E. S. Cassady and A. A. Oliner, "Dispersion relations in space-time periodic media" Proc. IEEEE, vol. 51, pp. 1342-1359, 1963 .

[3] B. R. Rao, "Electromagnetic wave propagation in a dielectric medium space-time modulated by a nonlinear pump wave." Proc. TEEE, rol. 56, pp. 1630-1631, 1968 .

[4] C. Elachi," "Electromagnetic wave propagation and source radiation in space-time periodic media," $\mathrm{Ph}$. D. dissertation, California Inst. Technol. 1971 .

[5] "D'ipole antenna in space-time periodic media," IEEE Trans. Antennas Propagat., vol. AP-20, pp. 280-287, May 1972.

[6] $\mathrm{L}$. M. Milne-Thompson, Calculus of Finite Differences. London. Fingland: Macmillan, 1933 .

\section{Comments on "The Relationship Between the Kirchhoff Approach and Small Perturbation Analysis in Rough Surface Scattering Theory"}

\section{Comments ${ }^{1}$ by Gaspar R. Valenzuela ${ }^{2}$}

In the above communication, ${ }^{3}$ Leader suggested that the scattering results by slightly rough theory [1]-[4] are a special case of those obtained by the Kirchhoff method of Beckmann and others [5], on grounds of being incorrect and without foundation.

By reasoning that the results for horizontal polarization and a slightly rough surface from both formulations are similar, which is a well known result, Leader concludes that the results for vertical polarization must also be similar, which is a most unique logic. The results for vertical polarization from slightly rough scattering theory are not in error, as he can check by himself by performing the algebra, and the difference of this result compared to that obtained with the Kirchhoff method is real.

The scattering results from the Kirchhoff method fail to explain the polarization dependence in the scattering of electromagnetic waves from rough surfaces of high conductivity in particular from the sea. Wright [3] in a wave tank experiment has verified the correctness of the perturbation results, which in first order are identical either by Rice method or by the method used by Wright. In first order the normalized cross section per unit area for horizontal

\footnotetext{
1 Manuscript received November 26, 1971

2 The author is with the Theoretical Studies Section, Electromagnetic Scattering Branch, Electronics Division, Naval Research Laboratory, Washington, D. C. 20390.

W. C. Leader. IEEE Trans. Antennas Propagat. (Commun.), vol. AP-20, pp. $786-787$, Nov. 1971 .
}

polarization is given by

$\sigma_{0 H}(\theta)=4 \pi \beta^{4} \cos ^{4} \theta\left|\frac{(\epsilon-1)}{\left[\cos \theta+\left(\epsilon-\sin ^{2} \theta\right)^{1 / 2}\right]^{2}}\right|^{2} W(2 \beta \sin \theta, 0)$

and for vertical polarization is given by

$$
\begin{aligned}
\sigma_{D V \nabla}(\theta)= & 4 \pi \beta^{4} \cos ^{4} \theta \\
& \cdot\left|\frac{(\epsilon-1)\left[\epsilon\left(1+\sin ^{2} \theta\right)-\sin ^{2} \theta\right]}{\left[\epsilon \cos \theta+\left(\epsilon-\sin ^{2} \theta\right)^{1 / 2}\right]^{2}}\right|^{2} W(2 \beta \sin \theta, 0)
\end{aligned}
$$

where $\beta=2 \pi / \lambda$ is the propagation constant of the incident plane wave in vacuo, $\theta$ is the angle of incidence of the propagation vector of the incident plane wave with respect the normal to the mean plane of the slightly rough surface, $\epsilon$ is the complex dielectric constant of the surface and $W(\cdot)$ is the two-dimensional wavenumber spectral density of the surface roughness (in Cartesian coordinates). Recent measurements on sea clutter demonstrate the correctness of these results in the context of the composite surface scattering model [6]-[8].

Contrary to what Leader has indicated for a perfectly conducting surface, i.e., $\epsilon=-i \infty$, (1) and (2) do become identical to the expressions obtained for a perfectly conducting surface, that is

$$
\sigma_{0 H H}(\theta)=4 \pi \beta^{4} \cos ^{4} \theta W(2 \beta \sin \theta, 0)
$$

and

$$
\sigma_{0 V V}(\theta)=4 \pi \beta^{4}\left(1+\sin ^{2} \theta\right)^{2} W(2 \beta \sin \theta, 0)
$$

and the convergence of the results to the perfectly conducting case was shown by Rice himself for horizontal polarization in his original paper.

The argument on the similarity of the reflection coefficients for both polarizations for a perfectly conducting surface has nothing to do with the backscatter case, except at normal incidence. In the specular direction Rice showed that if the spacing of the roughness was large compared with respect to the wavelength of the incident radiation the reflection coefficients were given by

$$
\begin{gathered}
R_{H H}=-1+2 \beta^{2} \cos ^{2} \theta\left\langle f^{2}(x, y)\right\rangle \\
R_{V V}=1-2 \beta^{2} \cos ^{2} \theta\left\langle f^{2}(x, y)\right\rangle
\end{gathered}
$$

where $f(x, y)$ is the surface roughness and the pointed brackets mean the ensemble average, also see Valenzuela [9]. Thus Leader in the arguments used to arrive at the objections, confuses the results for backscattering and for forward scattering.

Accordingly, since Leader has not really shown that the results by perturbation, and those by the Kirchhoff method for a slightly rough surface are the same, his arguments against depolarization [4] using the second-order terms in Rice's scattering formulation do not apply. However, we would like to indicate that multiplescattering is not the sole mechanism that yields depolarization. For example, Wright [10] and Valenzuela [11] have shown that a tilted slightly rough surface also depolarizes and volume scatter as pointed by Leader should also depolarize the incident electromagnetic radiation in many instances.

Measurements from the sea by Long [12] and Daley et al. [13] show that depolarization is very significant. For these cases probably only multiplescattering and the tilted plane mechanism are predominant.

Other recent investigations that also support Bragg scattering as the main mechanism responsible for sea clutter are those by Valenzuela and Laing [14], Hasselmann [15], and Wright and Keller [16].

We conclude indicating that Leader has not demonstrated that the scattering results by perturbation are a special case of those obtained with the Kirchhoff method for a slightly rough surface and experimental evidence on the contrary does support the predictions of slightly rough scattering theories. 